\title{
Quantum matter on a classical spacetime as a fundamental theory
}

\author{
André Großardt* \\ Queen's University Belfast, School of Mathematics and Physics, 2017 University Road, Belfast \\ BT7 1NN, United Kingdom \\ E-mail: a.grossardtequb.ac.uk
}

\begin{abstract}
The possibility of a theory in which only matter is quantised, and gravity is described by the classical theory of general relativity, even at the fundamental level, is not ruled out by any theoretical argument nor by experiment. Here, I discuss implications of this possibility and show that, from the most naive approach for such a theory, a gravity-matter coupling according to the semiclassical Einstein equations, one obtains a nonlinear Schrödinger equation as a non-relativistic limit that may be used to derive experimental predictions. Possibilities of experimental tests are discussed, which seem feasible with existing technology in the not-too-far future.
\end{abstract}

Corfu Summer Institute 2017 'School and Workshops on Elementary Particle Physics and Gravity'

2-28 September 2017

Corfu, Greece

${ }^{*}$ Speaker. 


\section{The gravitational field of quantum matter}

In 1975, Colella, Overhauser, and Werner [1] measured the phase shift induced by the Newtonian gravitational potential $V=-m g z$ on a neutron in a spatial superposition state. Their experiment is widely celebrated as the first observation of gravitational and quantum effects in a common system. For the more than four decades that have since passed, the experimental status of gravitational quantum physics has not changed much: the only observations performed involve external gravitational fields in their Newtonian limit.

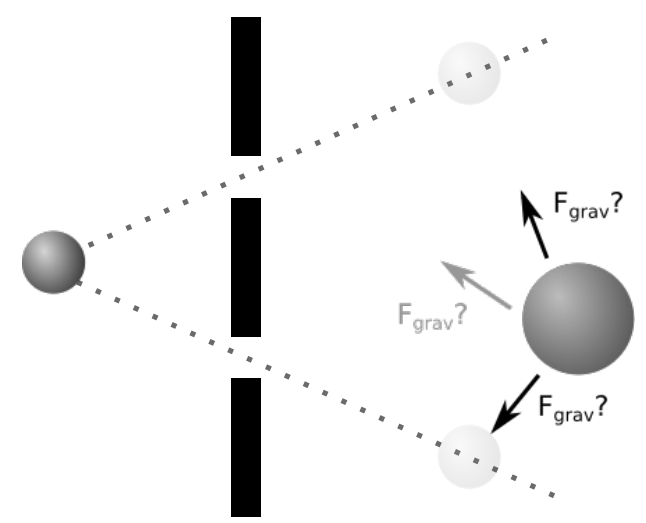

Figure 1: A massive quantum particle is found in a superposition state after having passed a double slit. What is the gravitational potential of this state, as sensed by a test mass?

The search for a unified theory that includes both gravity and quantum matter is driven by the opposite question: how quantum matter acts as a source of gravity. Arguably the simplest scenario to illustrate the problem is the double slit experiment, depicted in figure 1. If a massive particle passes a double slit, according to quantum mechanics, it will be in a superposition state of the possible paths. A second mass, which may very well be in a classical state, with a force sensor attached can serve as a device to monitor the gravitational potential. Assuming that we could create a superposition state massive enough, and a force metre sensitive enough, what gravitational potential would we observe?

It is important to remind oneself that whatever answer one comes up with is purely driven by theoretical ideas. There is no experimental evidence to back it up.

The default answer: quantised gravity Mainly inspired by the success of quantum field theory and the standard model of elementary particle physics, attempts have been made since the 1930s to reformulate general relativity as a quantum theory. The philosophical principle guiding most approaches seems to be that gravity is simply another field which should be quantised along the same lines as the quantisation of matter fields.

If the particle in figure 1 was electrically charged, then the electric field it creates after having passed the double slit would itself be in a superposition of two different field configurations. Similarly, from quantised gravity one would expect that the superposition of the massive particle results in a superposition of two different spacetimes.

However, as pointed out by Penrose [2], there is no well-defined way of comparing the timelike Killing vectors of both spacetimes to each other, and hence the quantum mechanical time evolution 
operator on such a superposition of spacetimes is fundamentally ill-defined. Consequentially, even in situations that are perfectly non-relativistic (velocities $v \ll c$ ) and well-approximated by Newtonian gravity ( $G m \ll R c^{2}$, where $R$ is the particle size) our current theories for quantum matter and for gravity are incompatible.

Usually, this incompatibility in the low energy regime is assumed to be resolved by perturbative quantum gravity (i.e. the quantisation of the metric field perturbation in linearised general relativity) which is seen as an effective theory for whatever the full quantum theory of gravity might be. The Newtonian gravitational potential for the superposed particle is then in a superposition, exactly as the electric potential for the charged particle would be. However, with the current state of quantum gravity research this assumption is little more than an educated guess. On the other hand, the nonrenormalisability of perturbative quantum gravity shows that gravity must be different from the standard model interactions in some respect.

The alternative: quantum matter on a classical spacetime The second possible solution starts with the assumption that general relativity provides a correct description of gravity, not just as the classical limit of some quantum theory, but in a fundamental sense; that the only thing that quantum physics changes is the way we describe matter fields living on spacetime-not spacetime itself.

While this idea may sound crazy at first sight, it is actually more conservative and closer to the intuition that lead Einstein to the discovery of general relativity. It is driven by an entirely different understanding of Einstein's theory: the lesson to learn from it is not that gravity is simply another field; rather, the main message is that there is no such thing as gravity —at least not as a fundamental interaction. There is only spacetime and the matter on it which both sources the curvature of spacetime and follows it with its motion.

Of course, these are only philosophical considerations which are no indication for the physical relevance of such an approach. In order to be taken seriously as a physical theory, one must address the questions whether there is a well-defined mathematical model consistent with past observations, as well as, which predictions it makes for possible future experiments. I will discuss the first question in section 2 and the second one in section 3 of this article.

\section{Fundamentally semiclassical gravity}

In general relativity, the curvature of spacetime is described by the Einstein tensor, defined from the metric tensor $g_{\mu \nu}$, the Ricci tensor $R_{\mu \nu}$, and the scalar curvature $R$ as

$$
G_{\mu v}:=R_{\mu v}-\frac{1}{2} g_{\mu v} R
$$

which forms the left-hand side of Einstein's field equations:

$$
G_{\mu v}=\frac{8 \pi G}{c^{4}} T_{\mu v}
$$

The right hand side of these equations is given by the classical stress-energy tensor of all matter fields. Although the matter in our universe is quantum, systems with a significant gravitational field are usually in a well-localised state $|\psi\rangle$, which is why the stress-energy tensor can be replaced by 
the expectation value of the corresponding quantum operator, yielding the semiclassical Einstein equations:

$$
G_{\mu \nu}=\frac{8 \pi G}{c^{4}}\left\langle\psi\left|\hat{T}_{\mu \nu}\right| \psi\right\rangle
$$

In quantised gravity one would expect this equation to break down if the states $|\psi\rangle$ becomes nonclassical, due to quantum fluctuations. However, if spacetime is assumed to be fundamentally classical, equation (2.3) provides one possible way to reconcile it with quantum matter fields. This possibility for a fundamentally semiclassical theory has been proposed already more than half a century ago $[3,4]$.

\subsection{Concerns about semiclassical gravity}

It is sometimes claimed that semiclassical gravity would be inconsistent, or even in contradiction to observation, often with reference to the works by Page and Geilker [5, 6] or by Eppley and Hannah [7]. Most of these claims are a variant of one of the following arguments:

Divergence freedom of Einstein's equations The Einstein tensor is covariantly conserved, i.e. it has vanishing four-divergence, $\nabla_{\mu} G^{\mu v}=0$, where $\nabla$ denotes the covariant derivative. Therefore, as a consequence of Einstein's equations, the stress-energy tensor must be covariantly conserved as well:

$$
\nabla_{\mu}\left\langle\psi\left|\hat{T}^{\mu v}\right| \psi\right\rangle=0
$$

However, when the state $|\psi\rangle$ is projected into one of the eigenstates of some Hermitian operator during a measurement, this equation is violated.

This, of course, only applies to the instantaneous collapse of the Copenhagen interpretation. In a no-collapse interpretation (e.g. many worlds), on the other hand, all branches will contribute to the gravitational field leading to obvious contradictions to everyday experience- - this is the main argument Page and Geilker [5, 6] illustrate with a simple torsion balance experiment.

The situation becomes more difficult if one adopts the point of view of an objective collapse of the wave function as in collapse models [8], and in fact it depends on the concrete way in which the collapse is introduced whether or not equation (2.4) is satisfied.

Superluminal signalling The second common concern against semiclassical gravity is the alleged possibility to violate causality, as it opens a possibility to signal faster than light. Essentially, the reason why superluminal signalling can occur is that semiclassical gravity gives access to the wave function itself, rather than expectation values of Hermitian operators. A concrete scheme for how to exploit this to send signals faster than light is depicted in figure 2. An entangled spin-state of two particles is created, with one particle each being sent to A and B, respectively, both of which have the ability to perform a spin-measurement using a Stern-Gerlach device.

In standard quantum mechanics, B will find a particle at positions $\pm d$ on the screen with $50 \%$ probability for each position, regardless of whether or not A measures the spin. With semiclassical gravity, however, the superposition state (figure 2, bottom) will gravitationally self-attract and show on the screen at positions $\pm d^{\prime}$ which are different from the positions $\pm d$ found for the collapsed state (figure 2, top). Therefore, from the measurement outcome at B one can determine whether or not A performed a measurement along the $z$-axis, and this decision (measurement yes/no) can be used to encode a binary signal. 


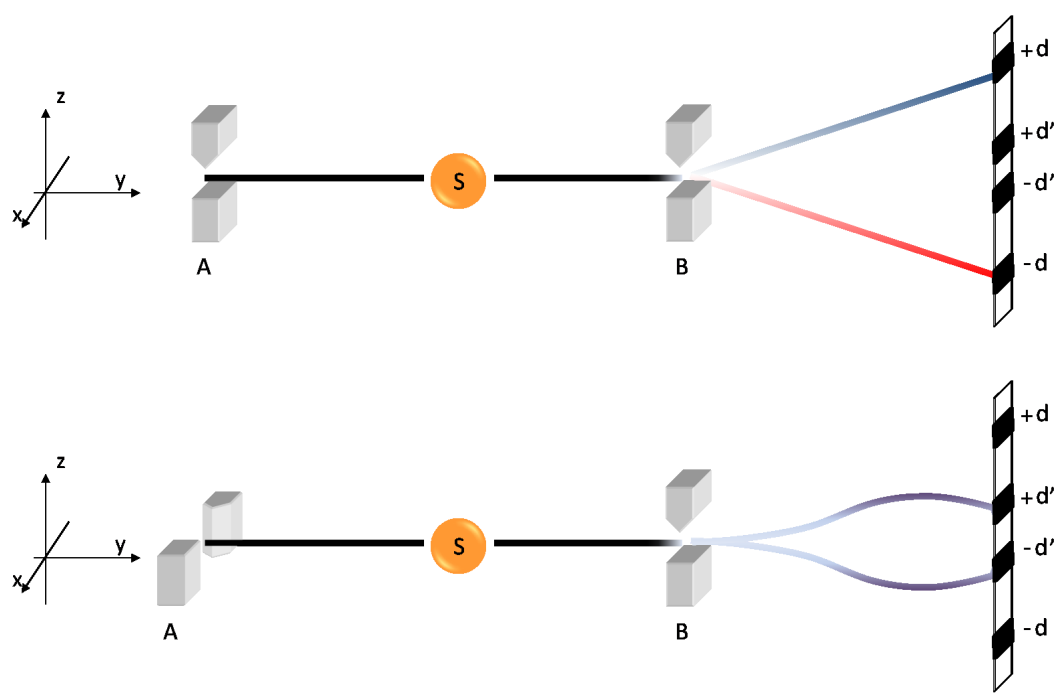

Figure 2: Predictions of semiclassical gravity for a pair of entangled particles passing through two distant Stern-Gerlach apparatuses [9]. While the state with well defined spin in $z$-direction measured by A (top) is found either around the position $+d$ or $-d$ with probability $50 \%$, the superposition state (bottom) where A does not perform a measurement in $z$-direction is subject to gravitational self-attraction and, therefore, is found either around the position $+d^{\prime}$ or $-d^{\prime}$. The measurement outcome at B can be used to infer the measurement basis ("signal") at A.

In principle, the distance $L$ between $\mathrm{A}$ and $\mathrm{B}$ does not matter, and if it is chosen such that $L>c \tau$, where $\tau$ is the time it takes to perform the measurements, then the signal will arrive at $\mathrm{B}$ faster than any light signal can. In practice, this is nearly impossible: even if one could measure the point of impact of a basketball sized sphere of osmium ${ }^{1}$ with the precision of one atomic diameter, and one could prepare a coherent superposition state of such a $170 \mathrm{~kg}$ object, A and B would still have to be more than $10000 \mathrm{~km}$ apart in order to actually communicate faster than light. Of course, the possibility of superluminal communication poses a potential problem even if it is not practically achievable. However, the huge gap between experimentally feasible parameters and the parameters required to exploit this possibility gives plenty of room for unknown effects (e.g. gravity induced wave function collapse) which may render this type of faster than light signalling impossible also in principle.

In addition, it is possible to introduce a nonlinear dynamics without permitting faster than light signals, namely to allow only for such nonlinear terms which depend on the local state and measurements in the past light cone [10,11]. In the case of semiclassical gravity, this would imply that after A's measurement the particle at B would still evolve as in figure 2 (bottom), and only after the information of the measurement at A reaches B (at the speed of light!) the evolution would change to the one in figure 2 (top). A collapse of this kind is completely compatible with standard quantum mechanics in the experimentally tested regime, and the nonlinearity could not be exploited for superluminal signalling. Although this collapse prescription makes predictions that differ from semiclassical gravity with the standard collapse in the case of entangled particles, there are no observable differences for single particle wave functions.

\footnotetext{
${ }^{1}$ With $\rho \approx 23 \mathrm{~g} / \mathrm{cm}^{3}$, osmium is the densest of the known elements.
} 
Unnatural collapse Finally, a third criticism concerns the applicability of semiclassical gravity for explaining the wave function collapse. Although it is introduced as a mere model to couple quantum matter to a classical spacetime, it would be a great feature if this non-quantum interaction could also resolve the measurement problem in quantum mechanics by leading to an objective collapse. The idea that the collapse may be caused by gravity has been proposed before $[12,13,2$, 14], however with different models.

In contrast to those stochastic models, semiclassical gravity describes a deterministic dynamics, which cannot explain the stochastic outcomes of measurement results in quantum theory (at least not without invoking some other source of stochasticity). A spatial superposition state $|\psi\rangle=\left(\left|\vec{x}_{1}\right\rangle+\left|\vec{x}_{2}\right\rangle\right) / \sqrt{2}$ of positions $\vec{x}_{1}$ and $\vec{x}_{2}$ would reduce ("collapse") to a narrow wave packet around the average position $\left(\vec{x}_{1}+\vec{x}_{2}\right) / 2$, rather than one of the two positions $\vec{x}_{1}$ or $\vec{x}_{2}$ with $50 \%$ probability each.

In conclusion, semiclassical gravity by itself seems incapable of explaining the wave function collapse, and some different collapse mechanism must be added to it in order to obtain a consistent theory. Nonetheless, there is no conclusive theoretical argument why this should not be the case, and therefore, the possibility of semiclassical gravity as a fundamental alternative to quantum gravity remains open.

\subsection{Alternative coupling schemes}

Equation (2.3) is only one possibility to couple quantum matter to a classical spacetime. In principle, different types of coupling are possible, as long as they agree with equation (2.3) in the classical limit. A specific proposal for an alternative coupling has been presented by Tilloy and Diósi [15], where the idea is to take an objective wave function collapse [8] seriously, and then use the collapse events described by this mechanism—rather than the wave function—as the source of the gravitational field.

From the mathematical description, this approach is equivalent to a weak measurement of the mass distribution, where the measurement signal is used as a feedback to determine the curvature of spacetime. As this scheme is mathematically equivalent to standard quantum mechanics with decoherence, there is no issue with superluminal signalling in this approach. This is ensured by adding stochastic terms to the semiclassical equation (2.3).

The collapse is driven by the stochastic process of the collapse model, and will lead to the common result of a superposition state ending up at each position with a certain probability, rather than in a deterministic average position. Of course, the origin of this stochastic collapse remains unknown in this approach, and it is unclear whether the resulting stochastic stress-energy tensor can be covariantly conserved.

\section{Newtonian semiclassical gravity and experimental tests}

\subsection{The Schrödinger-Newton equation}

In the Newtonian limit of non-relativistic velocities and weak gravitational fields, equation (2.3) becomes the Poisson equation

$$
\nabla^{2} V_{G}=4 \pi G\langle\psi|\hat{\rho}| \psi\rangle
$$


for the gravitational potential $V_{G}$, where $\hat{\rho}=m \hat{\phi}^{\dagger} \hat{\phi}$ is the mass density operator of a massive field with field operators $\hat{\phi}$. Inserting this potential into the second-quantised Schrödinger equation for $N$ particles yields the nonlinear $N$-particle Schrödinger-Newton equation:

$$
\begin{aligned}
\mathrm{i} \hbar \dot{\Psi}_{N}\left(t, \vec{r}_{1}, \ldots, \vec{r}_{N}\right) & =\left(-\sum_{i=1}^{N} \frac{\hbar^{2}}{2 m_{i}} \nabla_{\vec{r}_{i}}^{2}+V_{\text {linear }}+V_{G}\left[\Psi_{N}\left(t, \vec{r}_{1}, \ldots, \vec{r}_{N}\right)\right]\right) \Psi_{N}\left(t, \vec{r}_{1}, \ldots, \vec{r}_{N}\right) \\
V_{G}\left[\Psi_{N}\left(t, \vec{r}_{1}, \ldots, \vec{r}_{N}\right)\right] & =-G \sum_{i=1}^{N} \sum_{j=1}^{N} m_{i} m_{j} \int \frac{\left|\Psi_{N}\left(t, \vec{r}_{1}^{\prime}, \ldots, \vec{r}_{N}^{\prime}\right)\right|^{2}}{\left|\vec{r}_{i}-\vec{r}_{j}^{\prime}\right|} \mathrm{d}^{3} r_{1}^{\prime} \cdots \mathrm{d}^{3} r_{N}^{\prime}
\end{aligned}
$$

$\Psi_{N}$ is the $N$-particle wave function for particle coordinates $\vec{r}_{1}, \ldots, \vec{r}_{N}$, and $V_{\text {linear }}$ stands for any non-gravitational, linear potential. For the special case $N=1$ of a single particle, the SchrödingerNewton equation reads

$$
\mathrm{i} \hbar \dot{\psi}(t, \vec{r})=\left(-\frac{\hbar^{2}}{2 m} \nabla^{2}-G m^{2} \int \mathrm{d}^{3} r^{\prime} \frac{\left|\psi\left(t, \vec{r}^{\prime}\right)\right|^{2}}{\left|\vec{r}-\vec{r}^{\prime}\right|}\right) \psi(t, \vec{r}) .
$$

The gravitational potential is sourced by the wave function, as if its absolute value squared was a mass density, rendering the Schrödinger evolution nonlinear. This self-gravitational potential counteracts the free spreading of a wave packet according to standard quantum mechanics. The gravitational interaction decreases more slowly (with $1 / r^{2}$ ) than the quantum mechanical spreading (with $1 / r^{3}$ ) and, therefore, it can become comparable or even dominant for large masses and widely spread wave functions.

For elementary particles, however, the gravitational interaction is too weak to result in any observable effect ${ }^{2}$. In order to arrive at testable consequences of the Schrödinger-Newton equation one must study its behaviour for many-particle systems, where the evolution of the centre of mass is of particular interest.

Although, strictly speaking, the centre of mass motion cannot be separated in the nonlinear equation (3.2), an approximate equation can be obtained by taking into account that the mutual non-gravitational interactions that keep the many-particle system in its shape are much stronger than the gravitational terms. The gravitational potential in the centre of mass Schrödinger equation then takes the form of a triple convolution:

$$
V_{G}=-G \iiint \frac{\left|\psi\left(t, \vec{r}^{\prime}\right)\right|^{2} \rho\left(\vec{r}^{\prime \prime}\right) \rho\left(\vec{r}^{\prime \prime \prime}\right)}{\left|\vec{r}-\vec{r}^{\prime}-\vec{r}^{\prime \prime}+\vec{r}^{\prime \prime \prime}\right|} \mathrm{d}^{3} r^{\prime} \mathrm{d}^{3} r^{\prime \prime} \mathrm{d}^{3} r^{\prime \prime \prime}
$$

where $\rho(\vec{r})$ is the mass distribution of the constituents with respect to the centre of mass, which can be strictly defined in terms of the marginal distributions of the relative coordinate wave function ${ }^{3}$.

If the centre of mass wave function is much wider than the size of the system, the mass distribution $\rho(\vec{r})$ can effectively be considered point-like, and the Schrödinger-Newton equation takes the single particle form (3.3).

\footnotetext{
${ }^{2}$ For a proton, the gravitational interaction only becomes comparable to the free spreading for a wave packet size of about one million light years.

${ }^{3}$ see equation (37) of [16]
} 

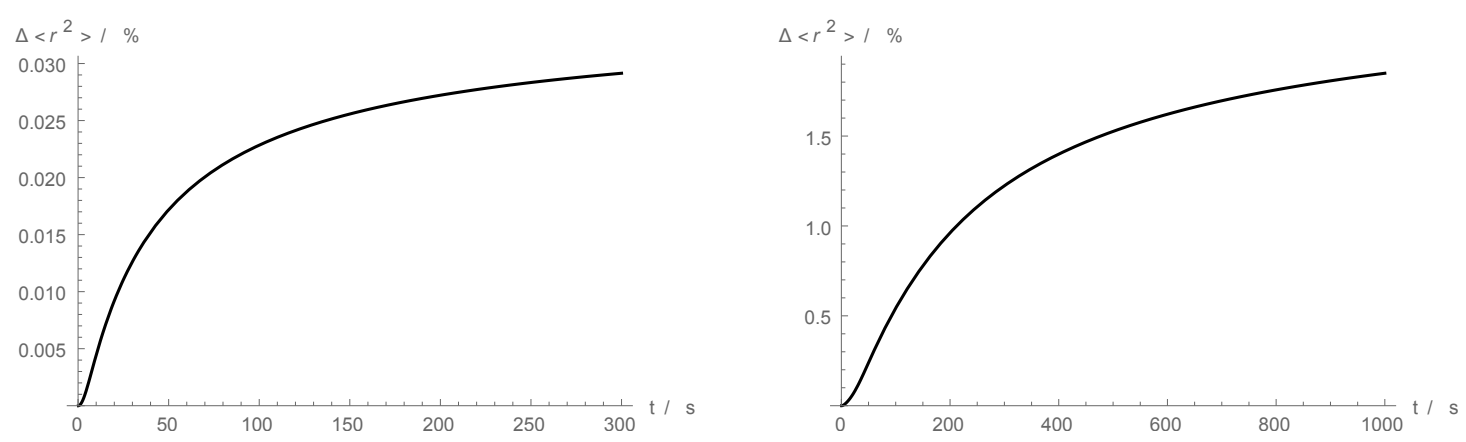

Figure 3: An osmium particle is initially trapped in a harmonic potential with frequency $\omega=0.1 \mathrm{~s}^{-1}$. The diagrams show the relative deviation of the wave function width according to the Schrödinger-Newton equation compared to the standard Schrödinger equation without self-gravitation. Left: for a mass of $10^{9}$ atomic mass units. Right: for a mass of $10^{10}$ atomic mass units.

\subsection{Experimental test of free spreading}

The free spreading of a spherically symmetric, Gaussian wave packet can be modelled under the assumption that self-gravitational effects are weak and, therefore, the Gaussian shape remains approximately intact:

$$
\psi(t, r)=\left(\frac{3}{2 \pi u(t)}\right)^{3 / 4} \exp \left(-\frac{3 r^{2}}{4 u(t)}\right),
$$

where $u(t)=\left\langle r^{2}\right\rangle(t)$ is the width of the wave function.

Generally speaking, for spherically symmetric wave functions, one can derive a set of first order differential equations for the second moments [17]:

$$
\begin{aligned}
\frac{\partial}{\partial t}\left\langle r^{2}\right\rangle(t) & =\frac{1}{m}\langle\vec{r} \cdot \vec{p}+\vec{p} \cdot \vec{r}\rangle(t) \\
\frac{\partial}{\partial t}\left\langle p^{2}\right\rangle(t) & =-m \frac{\partial}{\partial t}\left\langle V_{G}\right\rangle(t) \\
\frac{\partial}{\partial t}\langle\vec{r} \cdot \vec{p}+\vec{p} \cdot \vec{r}\rangle(t) & =\frac{2}{m}\left\langle p^{2}\right\rangle(t)-\left\langle\vec{r} \cdot \nabla V_{G}\right\rangle(t) .
\end{aligned}
$$

This is not a closed system, because the gravitational potential $V_{G}$ depends on the wave function. However, with the approximation (3.5) that the wave function remains a Gaussian, $V_{G}$ becomes a function of only the width $u(t)$ and the system can be cast into a third-order initial value problem:

$$
\dddot{u}(t)=-3 \omega_{\mathrm{SN}}^{2} f(u(t)) \dot{u}(t),
$$

with $\omega_{\mathrm{SN}}^{2}=G m / R^{3}$ for a sphere of mass $m$ and radius $R$, and the initial conditions

$$
u(0)=u_{0}, \quad \dot{u}(0)=0, \quad \ddot{u}(0)=\frac{9 \hbar^{2}}{2 m^{2} u_{0}}-\omega_{\mathrm{SN}}^{2} g\left(u_{0}\right) u_{0} .
$$

The functions $f(u)$ and $g(u)$ can be found in reference [17].

We can now solve equation (3.7) for different values of the total mass and initial spread of the wave function. Two exemplary results are shown in figure 3. For an osmium sphere of $10^{10}$ atomic mass units, initially trapped at $\sqrt{\left\langle r^{2}\right\rangle} \approx 1 \mathrm{~nm}$, we find that after 200 seconds $\left\langle r^{2}\right\rangle$ is about 
$1 \%$ smaller with the self-gravitational term than it should be according to the standard Schrödinger equation without self-gravity.

Experiments on earth do not allow for a free spreading time of 200 seconds - the particle would simply fall to the bottom of the vacuum chamber after a much shorter time span. However, satellite experiments [18] could reach the required parameters in terms of mass and detection accuracy.

\subsection{Experimental test with bound states}

Optomechanical systems have been considered as an alternative route towards testing the selfgravitational interaction according to the Schrödinger-Newton equation [19, 20]. This was first studied in the regime of a well localised wave function, hence, in the case where the width of the wave function $\psi(t, \vec{r})$ in equation (3.4) is much smaller than the width of the mass distribution $\rho(\vec{r})$. In this case, the Hamiltonian ${ }^{4}$ takes the effective form (omitting constant terms)

$$
H=\frac{p^{2}}{2 m}+\frac{m \omega_{0}^{2}}{2} x^{2}+\frac{m \omega_{S N}^{2}}{2}\left(x^{2}-2 x\langle x\rangle+\left\langle x^{2}\right\rangle\right),
$$

where $\omega_{0}$ is the frequency of the harmonic trapping potential, and $\omega_{\mathrm{SN}}$ characterises the strength of the self-gravitational interaction. For a homogeneous sphere of mass $m$ and radius $R$ we have, as before, $\omega_{\mathrm{SN}}^{2}=G m / R^{3}$. The nonlinearity of the Schrödinger-Newton equation is now encoded in the dependency on the position expectation values.

The self-gravitational term in the Hamiltonian (3.9) has consequences for the evolution of a squeezed Gaussian state [19, 21]. Although the evolution of the position expectation value is unaffected by the self-gravitational term and still satisfies the evolution equation

$$
\frac{\mathrm{d}^{2}}{\mathrm{~d} t^{2}}\langle x\rangle+\omega_{0}^{2}\langle x\rangle=0
$$

the evolution of the wave function itself is affected. The evolution of the variance of $x$ is now

$$
\frac{\mathrm{d}^{3}}{\mathrm{~d} t^{3}}\left\langle(x-\langle x\rangle)^{2}\right\rangle+4\left(\omega_{0}^{2}+\omega_{\mathrm{SN}}^{2}\right) \frac{\mathrm{d}}{\mathrm{d} t}\left\langle(x-\langle x\rangle)^{2}\right\rangle=0 .
$$

Hence, the internal oscillation of the wave packet is not in phase with its classical oscillation any longer, but there is a frequency offset

$$
\frac{\Delta \omega}{\omega_{0}}=\sqrt{1+\frac{\omega_{\mathrm{SN}}^{2}}{\omega_{0}^{2}}}-1 \approx \frac{\omega_{\mathrm{SN}}^{2}}{2 \omega_{0}^{2}} .
$$

An important insight, which significantly improves the feasibility of an experimental test, is that for a very well localised state (i.e. a very narrow centre of mass wave function) it is quite important to take into account that-for realistic matter-most of the mass density is localised around the position of the nuclei. The value of $\omega_{\mathrm{SN}}$ then depends on the atomic mass, as well as on the localisation $\sigma$ of the atoms within the crystal structure (given by the Debye-Waller factor): $\omega_{\mathrm{SN}}^{2} \propto G m_{\text {atom }} / \sigma^{3}$ with some pre-factor determined by the shape of the mass distribution. The numerical value depends only on the choice of material, with osmium having the most favourable

\footnotetext{
${ }^{4}$ For simplicity, we discuss only the one-dimensional case here, although all statements can be easily generalized to three dimensions.
} 


\begin{tabular}{lrrrr}
\hline Material & $m_{\text {atom }} / \mathrm{u}$ & $\rho / \mathrm{g} \mathrm{cm}^{-3}$ & $\sigma / \mathrm{pm}$ & $\omega_{\mathrm{SN}} / \mathrm{s}^{-1}$ \\
\hline Silicon & 28.086 & 2.329 & 6.96 & 0.096 \\
Tungsten & 183.84 & 19.30 & 3.48 & 0.695 \\
Osmium & 190.23 & 22.57 & 2.77 & 0.996 \\
Gold & 196.97 & 19.32 & 4.66 & 0.464 \\
\hline
\end{tabular}

Table 1: Atomic mass $m_{\text {atom }}$, mass density $\rho$, atomic localisation length $\sigma$, and characteristic frequency $\omega_{\mathrm{SN}}$ for selected elements.

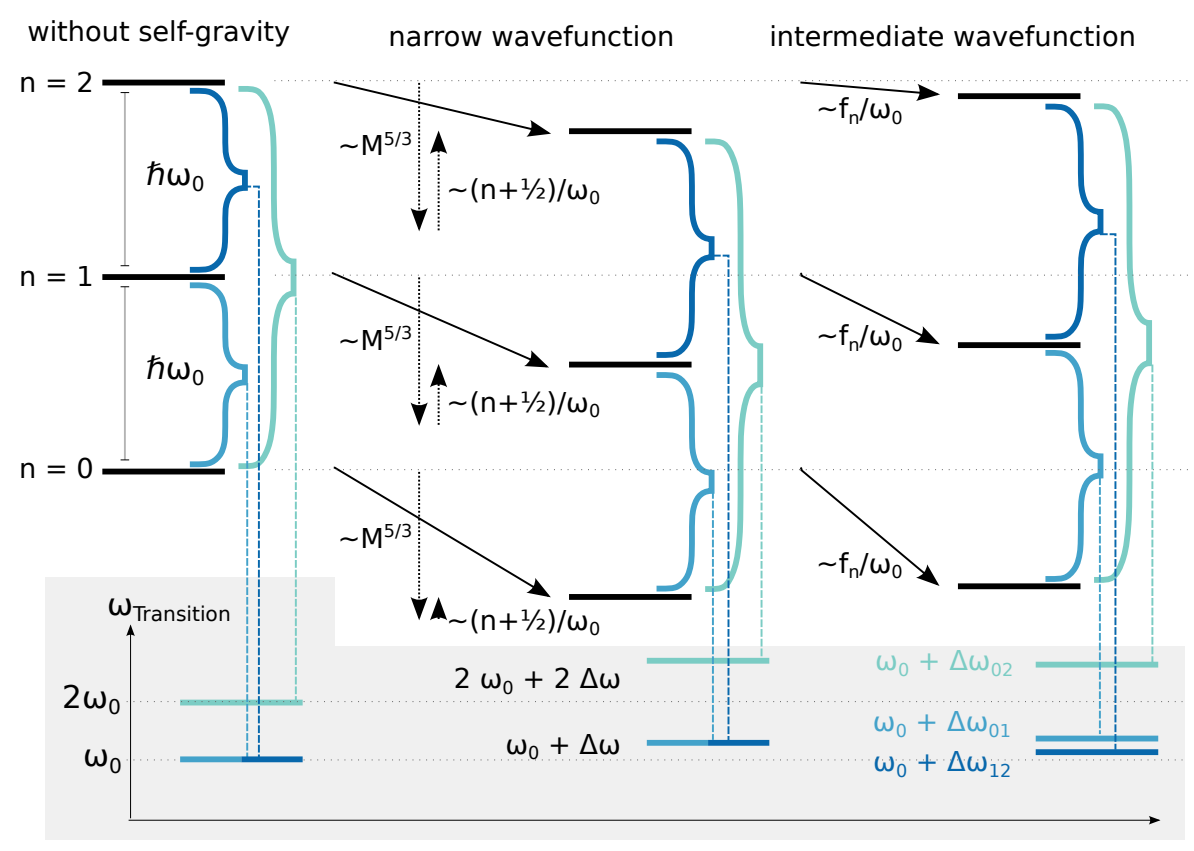

Figure 4: Effect of the Schrödinger-Newton equation on the spectrum [20]. The top part shows the first three energy eigenvalues and their shift due to the first order perturbative expansion of the SchrödingerNewton equation. The bottom part shows the resulting spectrum of transition frequencies. In the narrow wave function regime (middle part), all energy levels are shifted down by an $n$-independent value minus an $n$-proportional contribution that scales with the inverse trap frequency. In the intermediate regime, where the wave function width becomes comparable to the localisation length scale of the nuclei, this $n$-proportionality does no longer hold, leading to a removal of the degeneracy in the spectrum.

properties (cf. table 1). Experiments achieve a frequency resolution of up to $\Delta \omega / \omega_{0} \approx 10^{-6}$, implying that $\omega_{0}$ should be of the order of kilohertz or below for osmium particles or another order of magnitude lower for the experimentally well-approved silicon particles.

Note that, although this criterion is independent of the mass of the trapped particle, the requirement that the wave function be narrow compared to the particle size must be met, implying a minimum mass of the order of a nanogram.

Spectral effects The stationary states for the Hamiltonian (3.9) still display the same essential properties as the linear harmonic oscillator; specifically, although energy eigenstates are shifted, neighbouring energy levels all have the same distance to each other, whereas the quantitative 
change is too small to be detected (cf. middle column of figure 4).

However, the Hamiltonian (3.9) is only valid for a narrow wave function. If the wave function becomes comparable to the particle size, the shape of the wave function matters: a more localised wave function has a larger self-gravitational binding energy than a less localised one. This shape dependency leads to a different shift of the different energy levels in such a way that the energy gaps between neighbouring levels are not equal any longer. Consequentially, if the transition frequencies between different energy levels are monitored, the self-gravitational potential results in a fine structure, as is shown in the right column of figure 4.

Specifically, the shift $\Delta E_{n}$ of the energy levels can be calculated as first order perturbation, using the unperturbed harmonic oscillator eigenstates $\psi_{n}^{0}$, yielding the result:

$$
\begin{aligned}
\Delta E_{n}=\left\langle\psi_{n}^{(0)}\left|V_{G}\left[\psi_{n}^{(0)}\right]\right| \psi_{n}^{(0)}\right\rangle+\mathscr{O}\left(G^{2}\right) & \\
=-G m m_{\text {atom }} \sqrt{\frac{m \omega_{0}}{\pi \hbar}} \int_{0}^{\infty} \mathrm{d} \zeta & \exp \left(-\frac{2 m \omega_{0}}{\hbar} \sigma^{2} \zeta^{2}\right) P_{n}\left(2 \sqrt{\frac{m \omega_{0}}{\hbar}} \sigma \zeta\right) \\
& \times\left(\frac{\sqrt{2}}{\zeta} \operatorname{erf}(\sqrt{2} \zeta)-\frac{4}{\sqrt{\pi}}\right)+\text { constant term }
\end{aligned}
$$

The polynomials $P_{n}$ are defined as

$$
P_{n}(z)=\frac{\mathrm{e}^{-z^{2} / 2}}{\sqrt{2 \pi}\left(2^{n} n !\right)^{2}} \int_{-\infty}^{\infty} \mathrm{d} \xi \mathrm{e}^{-2 \xi^{2}} H_{n}(\xi)^{2}\left(\mathrm{e}^{2 z \xi} H_{n}(\xi-z)^{2}+\mathrm{e}^{-2 z \xi} H_{n}(\xi+z)^{2}\right),
$$

where $H_{n}$ are the usual Hermite polynomials. This result describes the spectral shift for a particle of mass $m$ trapped at frequency $\omega_{0}$, chosen such that the wave function width is comparable to the atomic localisation length scale $\sigma$ (cf. table 1). $m_{\text {atom }}$ is the atomic mass of the particle material, and the constant term in equation (3.13) refers to the state-independent contribution.

Figure 5 shows the resulting transition frequencies, i. e. the blue line (top) corresponds to the frequency for the transition between ground state and first excited state,

$$
\Delta \omega_{0 \rightarrow 1}=\frac{E_{1}-E_{0}}{\hbar}=\omega_{0}+\frac{\Delta E_{1}-\Delta E_{0}}{\hbar},
$$

whereas the yellow line (second from top) corresponds to the transition from first to second excited state,

$$
\Delta \omega_{1 \rightarrow 2}=\frac{E_{2}-E_{1}}{\hbar}=\omega_{0}+\frac{\Delta E_{2}-\Delta E_{1}}{\hbar},
$$

and so forth. One can see that for an osmium particle of nanogram mass, there is a significant spread of the transition frequencies, which opens a second possible path towards an experimental test. The main experimental advantage of this spectral analysis compared to the previously described dynamical effect is that no squeezed state needs to be prepared. A concrete proposal for an experimental realisation was presented in reference [20].

\section{Conclusion}

The fundamentally semiclassical approach to coupling quantum matter and gravity according to the semiclassical Einstein equations (2.3) provides us with an experimentally testable alternative 


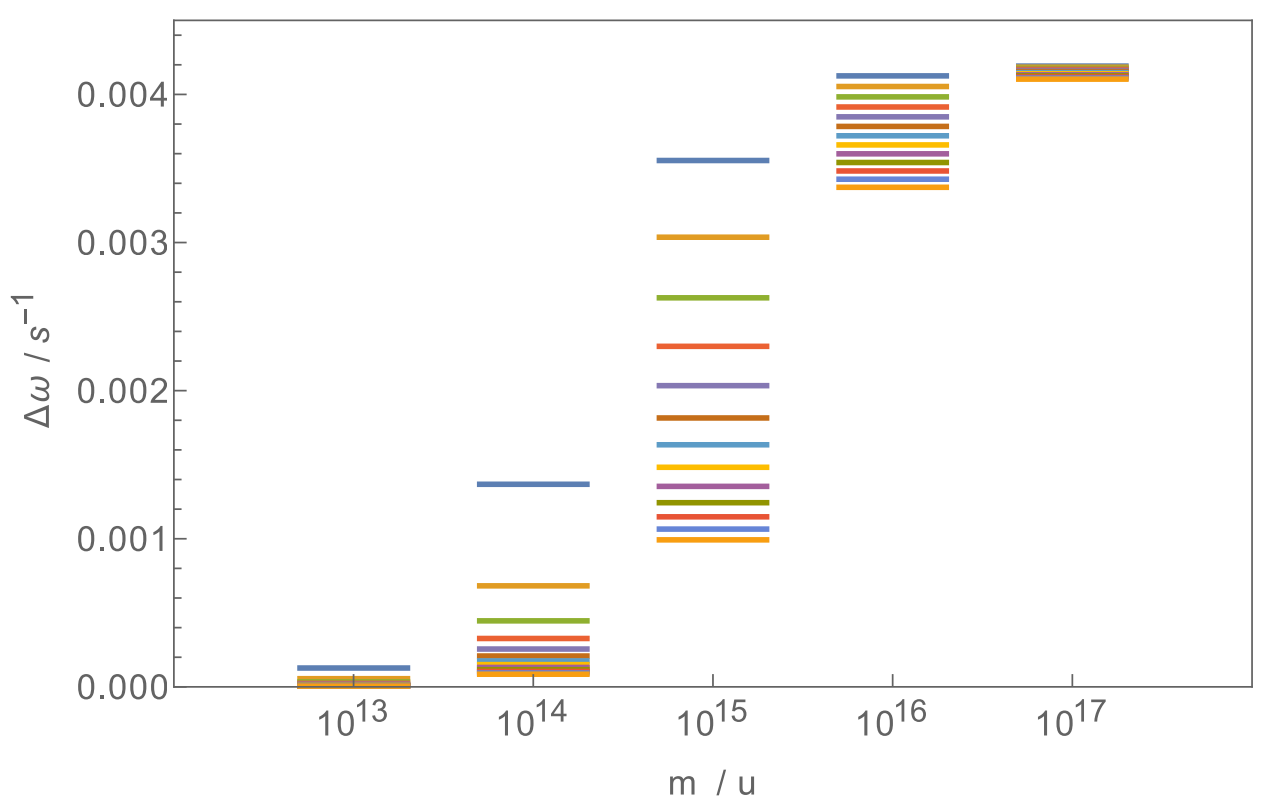

Figure 5: The frequency spectrum for the transition between neighbouring energy levels $(\Delta n=1)$ of a harmonic oscillator, for osmium at trap frequency $\omega_{0}=2 \pi \times 10 \mathrm{~s}^{-1}$. At low masses, self-gravity becomes negligible. At high masses all spectral lines are degenerate, shifted by the same constant $\Delta \omega$. The intermediate regime, where a significant splitting appears, spans about three orders of magnitude in mass [20].

to quantised gravity at low energies. Contrary to quantum gravity effects, the consequences of the semiclassical approach would show in experiments that are very close to being realisable in practice. As there is no conclusive theoretical argument against such an approach, these experiments are the only definitive way to rule out this fundamental model.

Unfortunately, ruling out the Schrödinger-Newton equation would not be conclusive evidence that the gravitational interaction must be quantised at low energies. Other coupling schemes than equation (2.3) could be possible, which may lead to different predictions in the non-relativistic regime. However, to date the only known alternative formulation of a consistent semiclassical model is the one by Tilloy and Diósi [15], which is based on collapse models [8] and, therefore, likely yields observable effects at similar mass scales as the Schrödinger-Newton equation.

\section{References}

[1] R. Colella, A. W. Overhauser and S. A. Werner, Observation of gravitationally induced quantum interference, Phys. Rev. Lett. 34 (1975) 1472-1474.

[2] R. Penrose, On gravity's role in quantum state reduction, Gen. Relativ. Gravit. 28 (1996) 581-600.

[3] C. Møller, Les théories relativistes de la gravitation, in Colloques Internationaux CNRS (A. Lichnerowicz and M.-A. Tonnelat, eds.), vol. 91, CNRS, Paris, 1962.

[4] L. Rosenfeld, On quantization of fields, Nucl. Phys. 40 (1963) 353-356.

[5] D. N. Page and C. D. Geilker, Indirect evidence for quantum gravity, Phys. Rev. Lett. 47 (1981) 979-982. 
[6] D. N. Page and C. D. Geilker, An experimental test of quantum gravity, in Quantum structure of space and time. Proceedings of the Nuffield Workshop, Imperial College London, 3-21 August 1981 (M. J. Duff and C. J. Isham, eds.), pp. 11-21. Cambridge University Press, Cambridge, 1982.

[7] K. Eppley and E. Hannah, The necessity of quantizing the gravitational field, Found. Phys. 7 (1977) 51-68.

[8] A. Bassi, K. Lochan, S. Satin, T. P. Singh and H. Ulbricht, Models of wave-function collapse, underlying theories, and experimental tests, Rev. Mod. Phys. 85 (2013) 471-527, [1204. 4325].

[9] M. Bahrami, A. Großardt, S. Donadi and A. Bassi, The Schrödinger-Newton equation and its foundations, New J. Phys. 16 (2014) 115007, [1407.4370].

[10] A. Kent, Nonlinearity without superluminality, Phys. Rev. A 72 (2005) 012108.

[11] B. Helou and Y. Chen, Extensions of Born's rule to non-linear quantum mechanics, some of which do not imply superluminal communication, J. Phys.: Conf. Ser. 880 (2017) 012021.

[12] L. Diósi, Gravitation and quantum-mechanical localization of macro-objects, Phys. Lett. A 105 (1984) 199-202.

[13] L. Diósi, Models for universal reduction of macroscopic quantum fluctuations, Phys. Rev. A 40 (1989) 1165-1174.

[14] S. L. Adler, "Gravitation and the noise needed in objective reduction models." 2014.

[15] A. Tilloy and L. Diósi, Sourcing semiclassical gravity from spontaneously localized quantum matter, Phys. Rev. D 93 (2016) 024026, [1509.08705].

[16] D. Giulini and A. Großardt, Centre-of-mass motion in multi-particle Schrödinger-Newton dynamics, New J. Phys. 16 (2014) 075005, [1404.0624].

[17] A. Großardt, Approximations for the free evolution of self-gravitating quantum particles, Phys. Rev. A 94 (2016) 022101, [1503.02622].

[18] R. Kaltenbaek, M. Aspelmeyer, P. F. Barker, A. Bassi, J. Bateman, K. Bongs et al., Macroscopic quantum resonators (MAQRO): 2015 update, EPJ Quant. Tech. 3 (2016) 5, [1503. 02640 ].

[19] H. Yang, H. Miao, D.-S. Lee, B. Helou and Y. Chen, Macroscopic quantum mechanics in a classical spacetime, Phys. Rev. Lett. 110 (2013) 170401, [1210.0457].

[20] A. Großardt, J. Bateman, H. Ulbricht and A. Bassi, Optomechanical test of the Schrödinger-Newton equation, Phys. Rev. D 93 (2016) 096003, [1510.01696].

[21] A. Großardt, J. Bateman, H. Ulbricht and A. Bassi, Effects of Newtonian gravitational self-interaction in harmonically trapped quantum systems, Sci. Rep. 6 (2016) 30840, [1510 . 01262]. 